\title{
Embedded Sanyog: a Portable Communication Tool for People with Multiple Disabilities
}

\author{
S. Dey \\ Dept. of Computer Science and Engineering, IIT Kharagpur \\ soumyad@cse.iitkgp.ac.in \\ R. Mukherjee \\ Dept. of Information Technology, ITME Kolkata \\ ratnajit101@yahoo.com \\ A. Basu \\ Dept. of Computer Science and Engineering, IIT Kharagpur \\ anupam@itkgp.ac.in
}

\begin{abstract}
Approximately 60 million people in India fall under the category of physically or mentally disabled out of which $\mathbf{2 . 1}$ million people are affected with severe speech and motor impairments (SSMI). Unfortunately only a minute fraction of them have any access whatsoever to Advanced Assistive Devices (AAD) which are still very expensive and beyond the affordability of a majority of people in India. Moreover, such devices generate text messages or speech mostly in English which makes them unusable by a majority of the Indian population. It should be our endeavour to make these assistive devices accessible to the common multitude. This paper briefly describes a portable version of Sanyog, an Augmentative and Alternative Communication(AAC) tool developed in India which caters to Indian multilingual needs. The communication tool is based on an iconic query-response interface. The system is currently available in English, Bengali and Hindi versions, and is used in a range of Indian institutions serving children with speech and motor impairments.
\end{abstract}

Keywords: Assistive Technology, SSMI, HCl, PDA, AAD, AAC

\section{INTRODUCTION}

Languages have evolved as the most vital form of communication among human beings, but in the process it divided humanity into mutually incomprehensible camps. Despite the fact that vision forms the most fundamental component of our perception, spoken and written languages have always dominated our communication process. However, for those with motor neuron and similar disabilities, these standard processes of communication do not really help. The Sanyog system aims at breaking the language barrier and making communication more natural for everyone through the development of visual languages. Gestures, body language, signs, symbols and of course icons are an integral part of our day-to-day communication, which we often tend to neglect, because they are so obvious. Out of these, icons seem to be a suitable candidate for developing a natural, economic and expressive communication medium between human beings across languages and between human and machines. As stated in [3] an icon is a representation of a concept, an object, an action or a relation. An icon can be interpreted by its perceivable form (word/syntax) or by the relation between its form and meaning. In this way icons also form a language, where each sentence is formed by a spatial arrangement of icons.

Although icon based assistive devices for SSMI patients are available in western countries since long, they are mostly tuned to the needs of western consumers as stated in [2]. By this, we mean that the icons in imported devices from US, UK and western Europe are tuned according to their socio-cultural scenario which differs remarkably from Indian socio-cultural scenario. As 
icon based communication requires SSMI patients to adapt to icons which depict concepts, imported devices contain icons that have little or no resemblance to the our environment. Unless these are lingua-culturally independent, they are of not much relevance in India. For example, let us consider the domain of cultural events or popular games. In both these domains, the representative items differ widely based on the social considerations. Moreover, the icon sets are never likely to be universal ones catering to everybody's requirement since that will make the icon selection process unnecessarily cumbersome. In this regard, an encouraging work has been the creation of a standard icon-set for basic communication requirements in the Indian context by the Indian Institute of Cerebral Palsy (IICP) based on detailed surveys and their teaching experiences. Hence, we take this icon set as a standard in our endeavour of generating (near) natural text and speech from icon selections.

However, the most important factor here is the language barrier. Imported devices can at most cater to a minuscule fraction of SSMI patients who can understand English or some other European language. Moreover the western AADs like Pathfinder, ChatBox and E-Z Keys needs to be imported and pricing is an issue. Thus an AAC system which specifically caters to the Indian lingua-cultural requirements was urgently required. Sanyog is the only icon based AAC system which takes input and produces output in Indian Languages e.g. Hindi, Bengali along with English.

The original desktop based Sanyog system was designed taking into account the lingua-cultural requirements of the Indian population. Hindi, being the Indian national language and Bengali, a language very popular all over eastern India were chosen from a plethora of languages commonly spoken across the country. The system allows the intended users to form natural language sentences through selection of iconic concepts, relevant to the Indian socio-cultural scenario. Thus the targeted users adapt well to the GUI, with minimal training and develop the necessary handling skills very quickly with some level of handling assistance from a human instructor. The automatically generated natural language sentences (in Hindi, Bengali as well as English) are fed to an Text to Speech System (TTS) called Shruti, to generate near natural audio output in all the three languages. A sample screenshot of the original system is given as below in Fig 1.

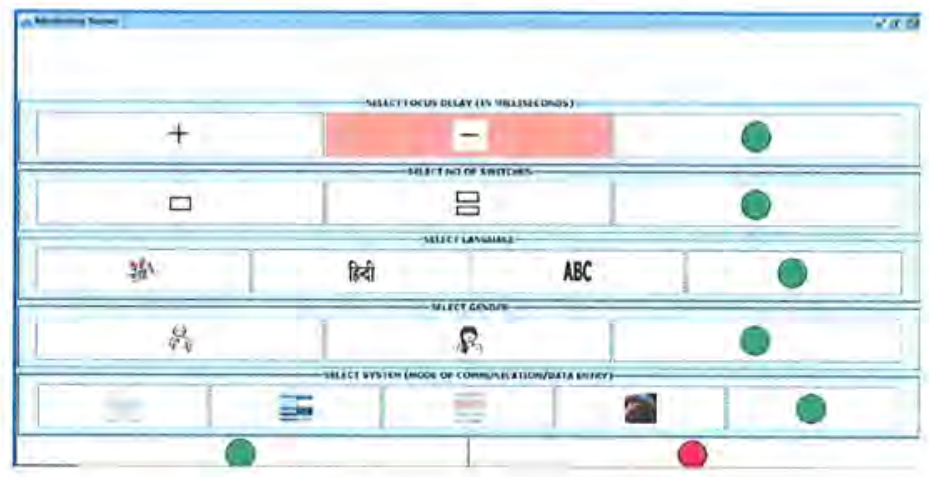

FIGURE 1: Sanyog Introductory Screen

However, a major drawback of the original system was the lack of portability, as desktop PCs cannot be wheelchair mounted. Laptops and netbooks are recent developments and are still costly enough, compared to the low end solution that is quintessentially targeted. Suggestions and feedbacks from the Indian Institute of Cerebral Palsy (IICP), where the system is currently in use and undergoing field testing have given us a clear idea that people with SSMI need a portable communication tool which should be light enough for mounting on their wheel chairs. With this motivation, the work on creating a portable version of the system was accentuated.

The current article is organized into the following sections. In section 2 we present a compact and terse overview of the original Sanyog system as to how it can be used by intended users for their daily communication. Section 3 highlights the various design issues that were formulated for the embedded version of the original system and the subsequent implementations. In section 4 we deal with the actual technology issues for porting Sanyog to handheld PDA platforms. In section 
5 we discuss scalability, compatibility and usability issues and finally we conclude the article in section 6 with probable future enhancements.

\section{OVERVIEW OF THE SYSTEM}

Human communication is a very delicate and convoluted process, involving various faculties of the human brain as explained in [1]. The process can be broadly divided into three sub processes:

- Concept accumulation relevant to a particular context/discourse.

- Natural language sentence generation from the concepts taking care of the syntax of the concerned language.

- Finally communicating them in any form speech or text messages etc.

For a majority of the people suffering with SSMI, these tasks are extremely difficult to perform. The core objective of our system is to assist such users in their communication needs. It has been found that icon based communication works very well with AAC users. The icons can be of varying complexities from relatively simple concepts like eating, running, sitting and objects like apple, fish, mango to relatively difficult ones like depicting a busy street, car accident, sword fighting etc. The iconic language supported by intelligent interpretation to words or phrases and eventually to meaningful sentences can be a solution to the above mentioned problem. A sample screenshot of the GUI helps in understanding the contextual hierarchy of icon selection as in Fig. 2.

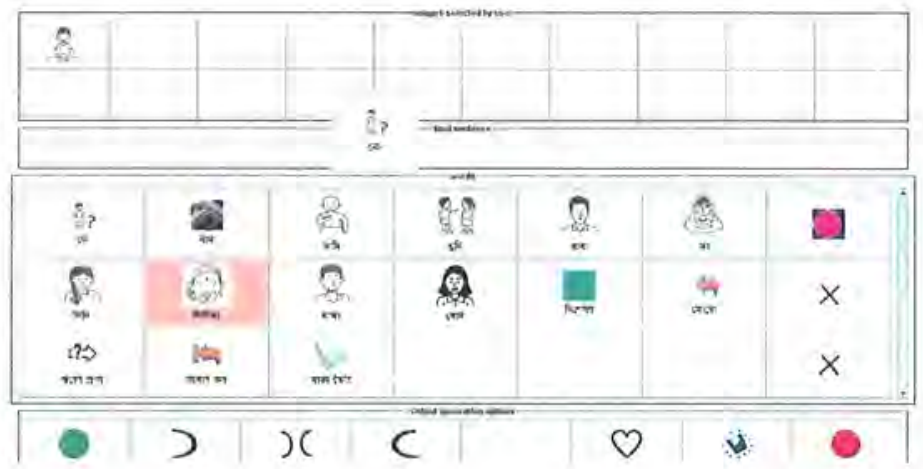

FIGURE 2: Sanyog Word Selection Panel

Sanyog is based on the above philosophy and developed as a communication tool for people suffering from SSMI. The salient features of Sanyog are:

- Accepts icon selection as user input, using mouse/stylus or special switches for people suffering from neuro-motor disabilities as they are unable to use the pointing device.

- Aimed at pre-literate children with speech and motor disabilities and based on Indian linguacultural icons and vocabulary. A point to be remembered in this context is that although such children may be unaware of sentence structures and components like verbs, nouns, adverbs etc, the system is essentially for iconic communication. Hence, even without a sufficient level of literacy, a user can easily generate a communicable sentence/speech by selecting appropriate icons ${ }^{1}$.

- Forms syntactically and semantically correct sentences in Indian Natural Languages like Bengali, Hindi and even in English.

- The generated sentences can be modified and inflected with mood and tense as per requirements.

- Finally the generated sentence can be spoken out by a TTS system thereby serving the purpose of communication.

'An icon for the verb 'run' will obviously have a picture of some human being running for easy understanding. 
The general architecture of the Sanyog system is broadly divided into few major modules:

1. Icon Selection: The system displays a set of icons organized in a hierarchy of contextual categories representing different concepts. These icons depict elements of a sentence like Verbs, Nouns, Adverbs, Qualifiers etc. The user has the choice of selecting multiple options from the different selection screens to express the concept(s) to be communicated. The current system has a selection interface which is verb directed. This means that the first screen in the icon selection panel shows a set of icons depicting different actions being performed (hence of type 'verb'). Following the selection of a verb ('eat' say), the system asks the user to select answers to different questions which essentially qualify the selected verb. For example, the next screen shows an icon for the question 'who' (represented by '?') with options like 'P, 'you', 'father', 'mother' etc in terms of icons of such choices². The user may select single or multiple options (depends on the what he/she intends to generate). Next comes the question 'what' with options like 'rice','soup' etc. Further, one can also select qualifiers of such objects like 'hot', 'cold' etc from a subsequent selection screen. ${ }^{3}$ Many other possible questions (when, where..) may also be answered by further selection depending on the user's requirement.

2. Natural Language Generation (NLG): After the user has selected all the concepts required to express his/her feelings, the NLG module forms natural language sentences in the chosen language inflected with mood,tense and person to form assertive, negative and interrogative sentences. The choice is again given by the user from appropriate selection screens. A sample selection trace by the user is given by

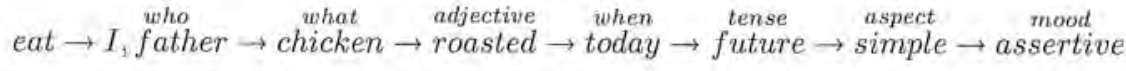

The trace exemplified above will output the following natural language sentence in English : 'Father and I will eat roasted chicken today'.

3. Text to Speech Synthesis: The text output is fed into the Indian Language TTS Shruti [6] for generating the corresponding speech output.

As we have said before that it is not necessary to understand the concepts of sentence formulation completely to use this system. As exemplified above, the system constrains the user to a specific mode of icon selection where the sole job of the user becomes the selection of the appropriate icon for the expression he/she is trying to communicate. The ordering of selection is taken care of by the system. Hence, the system is usable even by pre-literate children having little or no knowledge about sentence structure and grammar rules. A sample screenshot of the desktop system (with a sentence generated in Bengali) is given below in Fig. 3.

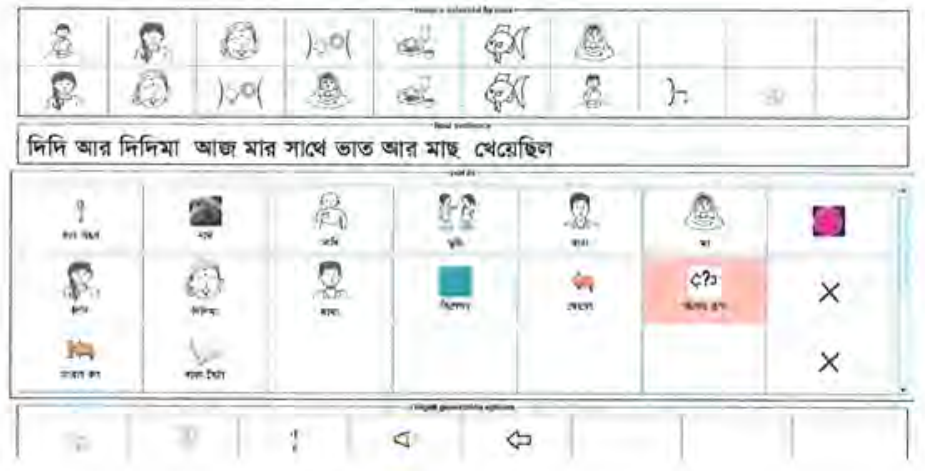

FIGURE 3: Sanyog System

\footnotetext{
${ }^{2}$ Here the role of an instructor becomes important. An icon for ' $I$ ' in the intuitive sense will be a person pointing him/herself. However, certain icons may not have such intuitive representations. Here the role of a standard (like the one by IICP) becomes important and the instructor enables the user to get acquainted with that standard.

${ }^{3}$ We can similarly have qualifiers for the verbs selected at the beginning.
} 


\section{DESIGN \& DEVELOPMENTAL ISSUES IN THE EMBEDDED VERSIONS}

The desktop version of the software developed in Java contains a lot of features which were equally adaptive to all kinds of users ranging from pre-literate to advanced. Though the desktop version can be ported to laptops and netbooks which far outperform any handheld devices in performance and compatibility, the primary issue regarding such implementations is the pricing. Even though the price of laptops and netbooks have plummeted in the recent years one still cannot find a decent laptop in India below Rs. 30,000/-(US\$600). Although some are available in the range of Rs. $20,000 /-($ US $\$ 400)$, they have numerous problems like build quality, battery backup etc. Netbooks are a very recent development and till date an Intel Atom powered netbook is not available in India below Rs.22,000/-(US\$440). Added to that neither laptops nor netbooks come packed with customized access switches and other access devices and it has been previously established in [1] that without these devices no system is of use to SSMI patients.

So considering both these class of devices is irrelevant, since they are not cost-effective enough. On the other hand PDAs cost less and they also provide a lot more battery backup than a standard laptop and thus are more suitable for portability. Standard handheld devices like WinCE preloaded smartphones and Linux based low-cost PDAs (like simputer and mobilis) are available at a range of Rs. $10,000-20,000 /-(U S \$ 200-400)$. Moreover, we already have the access switches made for the desktop version and we are in the process of modifying such switches (as shown in Fig 4) to make them compatible to handheld devices. However, portability and cost-effectiveness comes at the price of certain design constraints like:

- The computing power of handheld devices are much less compared to laptops and netbooks.

- The display size is usually in the range of 2.5 "-3.5" although some over 5 " are available, yet in comparison to the laptops and netbooks they are half or one-third the size.

- They run on operating systems which might not support all features available in desktop version.

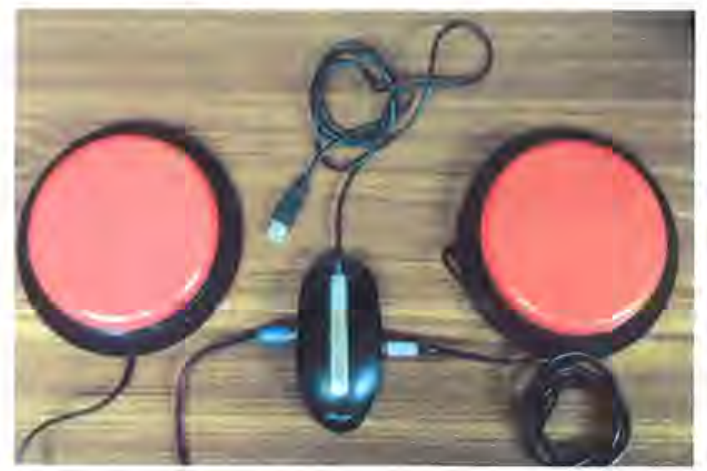

FIGURE 4: Access switches for desktop Sanyog system: the two switches are interfaced with a USB mouse for communication with the computer. This simple design decision helps in two ways: a) we do not need any additional circuitry for interfacing with the USB port b) the 'switch-press' actions can be easily captured by simple 'click-event' functions in the GUI.

The targeted users who are well accustomed to the desktop version might not accept a stripped down version of Sanyog. Thus it was an endeavour on our part to develop an equally feature rich compact edition of the original software (to the maximal possible extent) with integrated support for customized access switches.

In this section of the article we will discuss some of the problems and how those were bypassed to create a compact edition which is almost equally feature rich as the original edition. Infact some new features native to compact handheld devices are also being added in a way that it augments the capability of communication of the user. To carry out our task we decided to develop two 
different versions of the software which can be ported to both of 1. WinCE based and 2. Arm Linux based PDAs. Some of the basic hardware and software requirements are discussed below.

\subsection{Basic Hardware requirements}

- A touchscreen of form factor $3.5^{\prime \prime} \times 3.5^{\prime \prime}$ preferably.

- $\mathrm{CPU}$ speed $\geq 400 \mathrm{MHz}$

- $256 \mathrm{Mb}$ of RAM

- Secondary Storage $\geq 150 \mathrm{MB}$.

- 1 USB Port

- Special access switches developed for SSMI patients.

\subsection{Reasons for choosing WinCE}

- Windows CE is optimized for devices having minimal storage capacity. A WinCE kernel may run in under 1024 Kilobytes of memory.

- WinCE is a real-time operating system. The fundamental unit of execution is the thread which helps to simplify the interface and improve execution time of an application.

- WinCE has evolved into a component based, real-time, embedded operating system and is the primary platform in many portable devices.

- PDAs running on Microsoft WinCE offer a feature rich platform for deploying embedded applications and latest versions of Visual Studio support the development of WinCE based applications producing executable programs to run in an emulator or in an actual mobile device connected by a USB cable.

\subsection{Reasons for choosing Arm Linux}

- In the Linux-front, we created a version of the same application up and running on a Linux based embedded platform. The GUI was designed using the open source GTK library.

- Although still not very much common place, Linux based PDAs are regarded as one of the most potent competitors in the PDA market due to the royalty free operating system and growing maturity and easy-to-use GUI of the newly available kernels.

\subsection{Design Issues:}

- The net compact framework used for windows mobile application development offers limited features compared to the complete product suite used for desktop applications. This is the biggest obstacle in designing an attractive interface for WinCE based applications.

- For deployment on portable devices, there was a need to redesign the entire GUI optimizing it for the reduced screen size in a PDA. It is a challenge to fit all icons corresponding to a node in contextual hierarchy. The PDA version might not have all icons present in each icon selection stage as in the desktop version but we have tried to fit in as many as possible (both in WinCE and arm-Linux).

\subsection{Developmental issues:}

- PDAs are originally designed to be operated by stylus (provided with the device) but it is almost impossible for SSMI patients to operate a stylus. So a special switch access mechanism has to be developed for them to operate the PDA.

- For desktop based systems, we have special access switches customized according the user's requirement (touch switch, press switch, lip/palm operated switch etc). However, such switches rely on serial-port based communication, a mechanism absent in PDAs. Hence, the special access mechanisms developed for the desktop has to be reconfigured to connect via USB port(s). This is still an ongoing work.

- The internal data structures for handling the image loading and natural language generation capabilities have also been entirely re-worked for smooth running in devices with reduced clock rates and memory as compared to PCs.

- The Indian language TTS shruti has been cross-compiled and ported to both WinCE and arm-Linux systems for generating the speech output. 


\section{GENERAL MODIFICATIONS AND ENHANCEMENTS FOR PDA VERSIONS}

As stated previously, the original desktop version had to be extensively modified due to design constraints, so as to optimize them for the small screens. Some of the general modifications and enhancements are discussed in the following sub-sections.

\subsection{Hardware and Software used for PDA version}

- Device: Samsung SGH i900 smartphone (WinCE), Simputer and Mobilis (arm-Linux).

- Operating System: WinCE 6.1 Mobile, arm-Linux.

- Development Framework: Microsoft.NET compact framework 2.0 for Visual C\#.net 2005 (WinCE), GTK library for C based GUI (Linux).

\subsection{Modifications and Enhancements in PDA Version}

The PDA version has been so designed that it takes advantage of the screenwidth and screenheight of a given device and accordingly adjusts the GUI. Some of the modifications and enhancements are:

- Dynamic numbers of panels as per number of icons per screen.

- Each panel contains maximum 4 pictureboxes for displaying 4 icons side by side spanning throughout the screenwidth of the display as seen in Fig. 4.2. This was done to preserve neatness throughout the screen e.g. For 20 icons we have 5 panels one below other, each panel containing 4 icons.

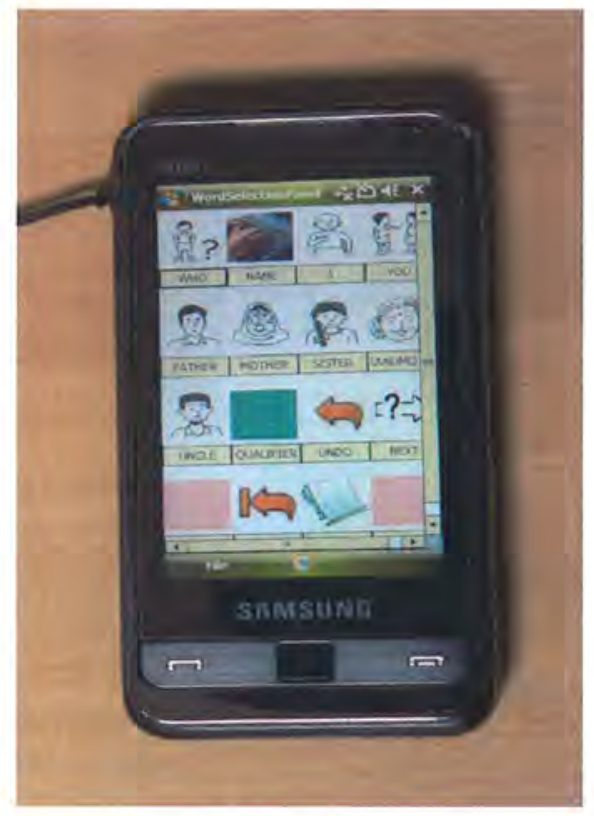

FIGURE 5: Embedded Sanyog Word Selection Screen

- A button has been placed below each picturebox to display the text corresponding to that icon and for click control.

- The autoscanning function automatically scans the panels one by one. On selection in a particular panel, the individual picture boxes are scanned in a cyclic order. When a picturebox is brought into focus by the autoscanner, the corresponding button's background color is changed to red which symbolizes focus.

- On selection of a particular picture/icon, the corresponding node is noted by intelligent icon interpretation and stored in a text file. The autoscanning and intelligent icon interpretation is almost similar to that of the desktop edition with some trivial modifications. 
- After taking the input from the word selection screen, the user chooses the corresponding tense, mood and type of sentences from a mood-tense panel. This feature gives the NLG the required mood-tense inflection to generate a grammatically correct sentence.

- Finally after selection of mood, tense and type of sentence (Assertive/Negative/Interrogative), the control shifts from mood-tense panel and the input is fed into the NLG. The NLG in response generates the final sentence in corresponding English or Bengali as chosen in the outset.

\subsection{Mode Sensitive GUI}

The access switch mechanism as shown in Fig. 4 operates in two distinct modes: single switch and two switch. Presently, the system requires that the mode should be specified at the outset. Depending on the access mode selected, the system adapts the GUI where the objective is the ease of navigation. As the number of switches reduce, more computation is pushed towards the system end.

- If the user selects single switch option, then the GUl reverts to autoscan mode, and a switch click indicates icon selection.

- In 2 switch mode, one switch is used for navigation and the other for selection. The GUI is scanned row-wise on click of the navigation switch. On selection of the row, column-wise icon scan of the selected row starts. The icon-element in that row can be selected by the selection switch. A sample screenshot of the Introductory panel and mood selection panel in Embedded Sanyog is given in Fig. 6.

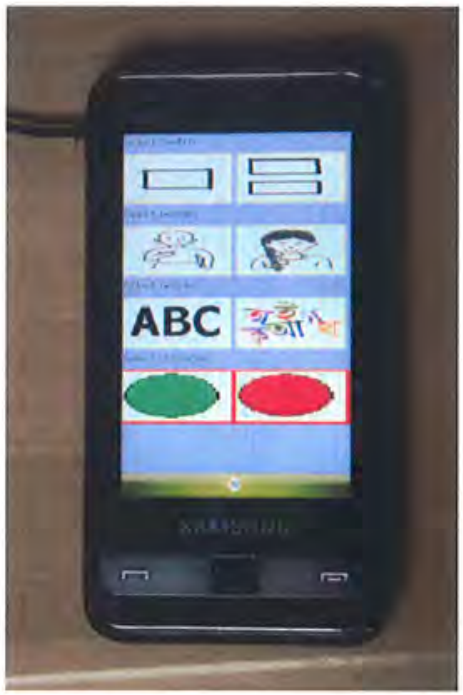

(a) Embedded Sanyog Introductory Panel with mode selection options (languages, access mode : 1 or 2 switch, gender)

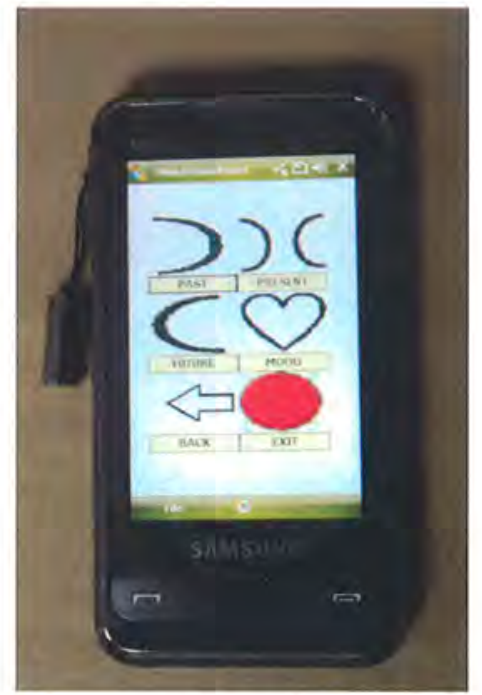

(b) Embedded Sanyog Mood-Tense selection panel

FIGURE 6: Embedded Sanyog: different control panels

The navigation time is indirectly proportional to the number of access switches used. More the number of access switches, less the computation the software performs and less will be the navigation time.

\section{PORTABILITY, SCALABILITY AND USABILITY ISSUES}

Portability: Essentially the PDA version is an abridged edition of the desktop version but we are trying to incorporate all the features of the original desktop version. It can be improved to make it 
nearly as capable as the desktop version by incorporating the Iconic System, SMS Application (for messaging the generated text) and Predictive Keyboard (a scanning based keyboard for advanced and literate users) all into one grand system which will be almost functionally similar to the desktop version but much more portable.

Scalability: Some of the systems which are functionally more suited and inherent to the desktop edition like a learning system (a trie based dictionary for prediction of user selection) and increasing icon repository etc. will not be incorporated in PDA versions due to memory restrictions.

Usability: It has been stated before that the system is developed without any assumption of the users knowledge of the communication language. Thus the GUI has been optimized in a way that icon selection is driven in a specific order and the system is able to get sufficient number of inputs from the user to generate the desired sentence. The NLG engine generates syntactically and semantically correct sentences abiding by grammatical rules which is true in both Indian and English languages.

\section{FUTURE ENHANCEMENTS}

- Presently the access device will communicate to the PDA through USB ports only. But if it can even be extended to use infrared port or bluetooth, then it will significantly reduce the hassles in carrying a mesh of cables everywhere.

- Predictive Keyboard: The Predictive keyboard unlike the standard "QWERTY" keyboard is a special keyboard equipped with automatic scanning function and word prediction system such that it can be used by SSMI patients. Advanced users with more cognitive abilities use this functionality frequently for communication purpose. This keyboard enables them to write a complete syntax without touching the icon interface at all and produces more customized outputs. The predictive keyboard for the PDA version has to be entirely reworked and optimized for small screen devices.

- SMS Application: One of the inherent advantages, native to handheld systems, like WinCE smartphones are the capability sending text messages. By augmenting a text messaging system with this version, it will be possible to send messages in both English and Bengali. The SMS application can be independently attached with the iconic interface as well as the predictive keyboard so that they individually act as independent system(s). This application can be augmented with a built-in autoscanning numberpad which helps the user to enter in the number to which he/she intends to send the SMS. An icon based phone book using which a user can send SMS to some pre-stored numbers (the pictures of the receivers are used as icons) is also being conceived.

- Till date the iconic interface has been developed for English and Bengali. The Hindi version along with the customized Text to Speech system for all languages is still in early developmental stages.

In short, the ongoing work is focused on developing a predictive keyboard and SMS application system in both English and Bengali.

\section{CONCLUSION}

In this paper an overview of the work, carried out at IIT Kharagpur on portable assistive devices for people with neuro-motor disorders has been presented. However there is always scope for more refinements and improvements. Its a noble issue to develop similar applications for a wide array of platforms, such that the reachability of this application increases and more people are benefitted. 


\section{ACKNOWLEDGEMENT}

This work was carried out in Communication Empowerment Laboratory, IIT Kharagpur as part of the Sanyog-ll project funded by Media Lab Asia. The authors further acknowledge the efforts of Mr. Samit Patra (Electrosoft Consultants, IIT Kharagpur) for moulding and supplying the access switches.

\section{REFERENCES}

[1] Bhattacharya S., Sarkar S. and Basu, A. Sanyog-A Speech Enabled Communication System for the Speech Impaired and People with Multiple Disorders. Journal of Technology in Human Services (JTHS). PA: Haworth Press. Vol. 25, Issue-(1/2), pp. 177-180, 2007. .

[2] Basu, A., Sarkar, S., Chakraborty, K., Bhattacharya, S., Choudhury, M. and Patel, R. Vernacular Education and Communication Tool for the People with Multiple Disabilities. Development by Design conference (dyd02). Bangalore, India.

[3] Bhattacharya, S., Sarkar, S. and Basu, A. Speech Enabled Communication Tool for the Speech Impaired and People with Multiple Disorders. Human Services Information Technology Applications (HUSITA 7th: 2004: Hong Kong, China)

[4] Fitrianie, S. and Rothkrantz, Leon J.M. An Icon-Based Communication Tool on a PDA, Euromedia 2005, ISBN 90-77381-17-1, no. 11, pp. 83-90, April 2005.

[5] Fitrianie, S. and Rothkrantz, Leon J.M. Language-Independent Communication using Icons on a PDA, TSD, September 2005.

[6] Mukhopadhyay A., Chakraborty S., Choudhury M., Lahiri A., Dey S. and Basu A. Shruti An Embedded Text-to-Speech System for Indian Languages, IEE Proceedings on Software Engineering - 153(2), pp. 75-79, April-2006. 